\title{
REPARTITION DES SIMULIIDAE (DIPTERA) DANS LE LOT
}

\author{
par J. GAGNEUR'1.
}

\begin{abstract}
Quatorze espèces de Simuliidae ont été capturées dans le Lot et ses affluents. Les biotopes, les préférences écologiques, la répartition altitudinale, la période de vol et les espèces associées sont précisées pour chaque espèce. L'étude quantitative de la distribution des larves a permis un essai de zonation. Le genre Prosimulium domine au dessus de $1000 \mathrm{~m}$, tandis que $O$. variegata a son maximum plus bas, jusqu'à $350 \mathrm{~m}$. Wilhelmia equina et $W$. salopiensis se rencontrent de préférence dans l'hy'porhithron. Dans la partie canalisée le nombre d'espèces est très réduit. Seule $S$. argyreatum est abondante dans le potamon.
\end{abstract}

\section{Distribution of Simuliidae (Diptera) in the River Lot.}

Fourteen species of S:muliidae have been taken in the Lot and its tributaries. The biotopes, ecological preferences, altitudinal distribution, flight period and associated species are given for each species. The genus Prosimulium is dominant at an altitude greater than $1000 \mathrm{~m}$, whilst $O$. variegatus is most abundant at a lower altitude up to $350 \mathrm{~m}$. Wilhelmia equina and $W$. salopiensis are chiefly found in the hyporhithron. The number of species is very reduced in the canalised section of the Lot. Only $S$. argyreatum is abundant in the potamon.

\section{INTRODUCTION}

La distribution des Simulidés a été étudiée en divers cours d'eau français : Dorier et Freychet (1945, 1948), DorIer (1963), Grenier (1949) pour les Alpes, Grenier et Bertrand (1951), Neveu (1972), Lavandier (1976) pour les Pyrénées, Giudicelli (1961) pour la Corse, SERRA-Tosio (1963) pour le bassin de l'Ardèche.

Le présent article porte sur les peuplements en simulies du bassin du Lot, sur le versant Ouest du Massif Central ; les renseignements obtenus sont utilisés pour élaborer une zonation amont-aval des cours d'eau de cette région.

1. Bellefontaine, 2851 Ocourt, Suisse.

Je tiens à remercier ici M. B. Serra-Tosio de Grenoble pour son aide dans la détermination des Simuliidae et ses conseils pour la rédaction de ce travail. 


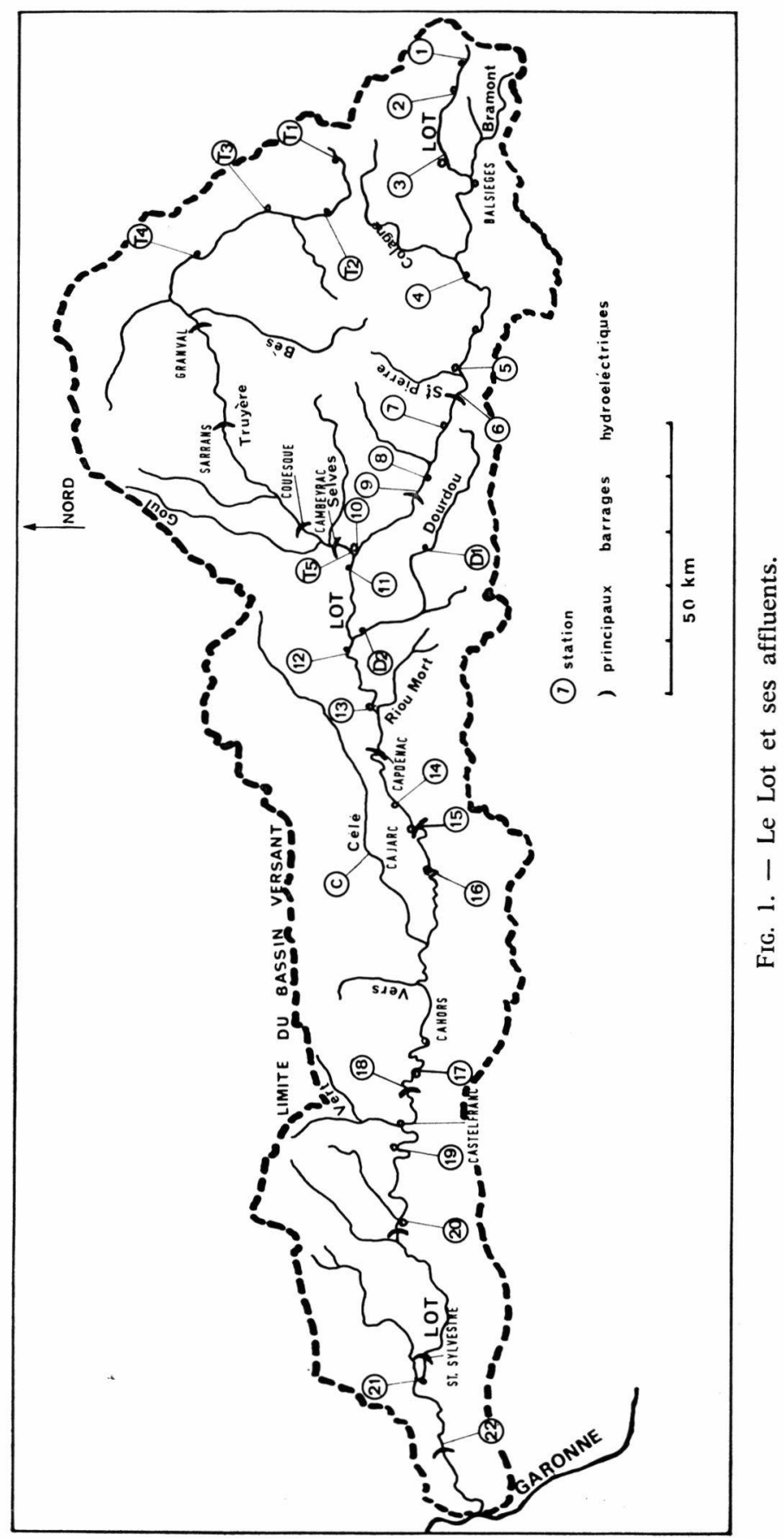




\section{1. - STATIONS ETUDIEES}

Le Lot, affluent rive droite de la Garonne, prend sa source à $1295 \mathrm{~m}$ dans les Monts Lozère et se jette dans la Garonne à Aiguillon après un cours de $491 \mathrm{~km}$. Au total, 30 stations, réparties sur le Lot et ses principaux affluents (Truyère, Dourdou, Célé) ont été étudiées.

La figure 1 donne l'emplacement des stations.

Le lecteur se reportera aux travaux suivants pour une description détaillée de la rivière : BoRdes, LucchetTa et Rochard (1973), BERthélemy et Laur (1975), Dauta (1975), Décamps, Massio et Darcos (1976).

\section{2. - METHODES}

Les prélèvements ont été effectués de décembre 1972 à juillet 1974, à raison d'un par mois et par station. La faune benthique, récoltée à l'aide d'un filet de vide de maille de $0,5 \mathrm{~mm}$, est conservée dans du formol à $\mathbf{4} \%$. Les imagos sont capturés au filet d'entomologiste. Les larves et les nymphes de Simuliidae sont récoltées sur les pierres et les plantes aquatiques à l'aide de pinces et d'une lame de couteau. A chaque station, l'échantillonnage a été fait pendant un temps variant de 5 à 10 minutes.

\section{3. - LE PEUPLEMENT SIMULIIDIEN DU BASSIN DU LOT}

14 espèces de Simuliidae ont pu être recensées. Les larves et les nymphes ont été déterminées d'après les travaux de GrenIER (1953), Knoz (1965), Podszuhn (1967), et Davies (1968).

Les données écologiques, la répartition altitudinale (fig. 2), et les dates d'émergence sont fournies pour chaque espèce quand cela est possible. Les dates d'émergence sont données d'après le nombre d'exuvies nymphales rapporté au nombre de nymphes. Les déterminations d'imagos, souvent difficiles, n'ont été utilisées que pour confirmation.

1) - Prosimulium hirtipes (Fries.) : cette espèce se rencontre en altitude, au dessus de $700 \mathrm{~m}$. Elle est abondante dans les ruisselets froids à fond rocheux ou sur la végétation aquatique. Emergences : VI-VII.

2) - Prosimulium nigripes End. = P. tomosvaryi End. La synonymie a été établie par ZwIck (1974). Cette espèce, sténotherme d'eau froide comme la précédente (GRENIER 1953, RUBzov 1959-1964), a 
son maximum d'abondance au-dessus de $1000 \mathrm{~m}$ dans le Lot et La Truyère. VII.

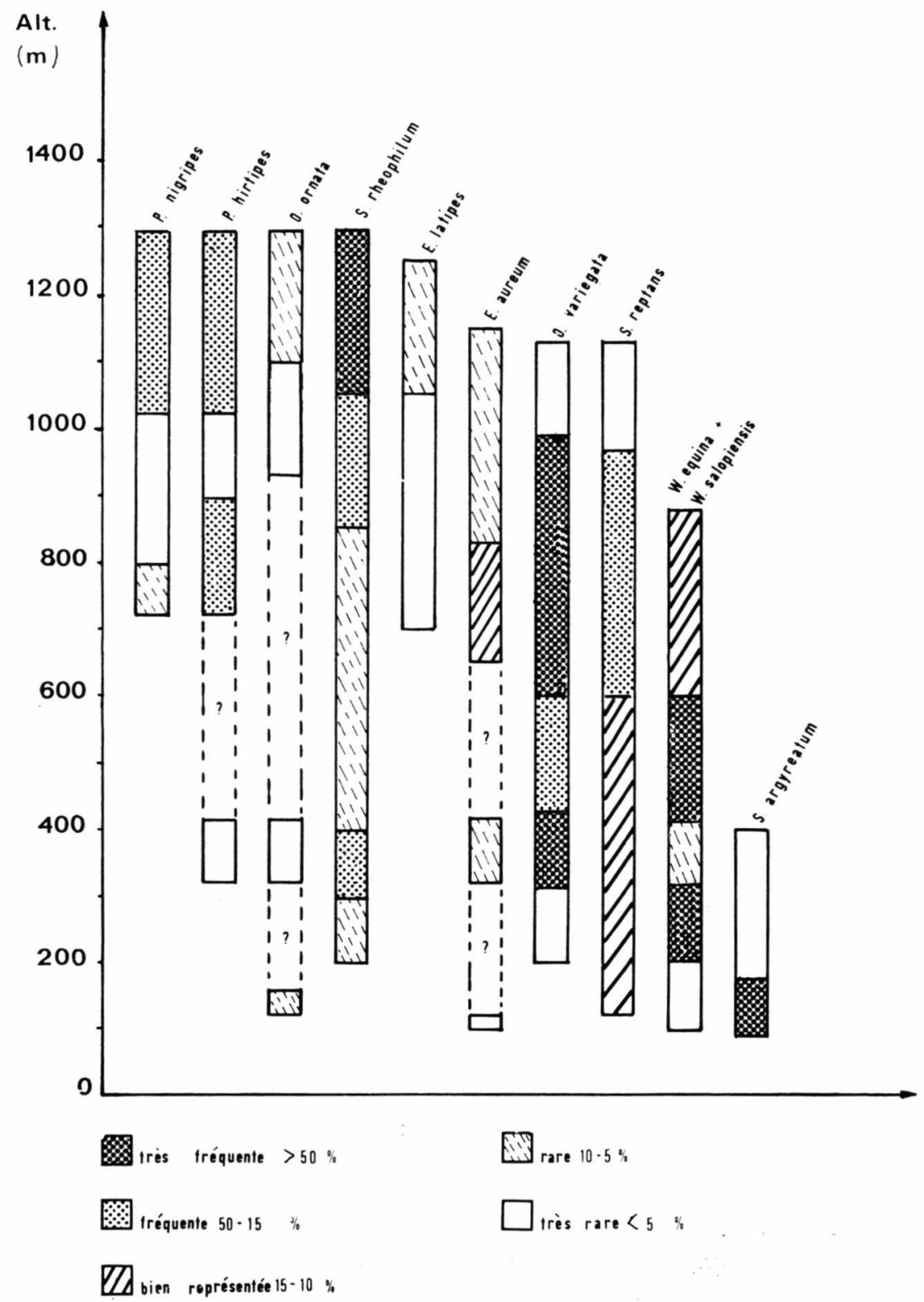

FIg. 2. - Répartition altitudinale des Simuliidae du bassin du Lot. 
3) - Prosimulium rufipes (Mg.) : peu abondante, elle n'a été récoltée qu'à Mende et dans le Dourdou.

4) - Eusimulium aureum Fries. : RrvosecchI (1962) montre qu'il s'agit d'un groupe d'espèces. Ceci explique les opinions divergentes de plusieurs auteurs (Grenier 1953, Dorier 1963, Rubzov 1959-1964). En effet, dans le Lot cette espèce se rencontre dans les ruisselets de sources froides et aux déversoirs de biefs (Luzech), là, où l'eau a une tendance nette à l'eutrophisation. Les éclosions ont lieu en juin-juillet pour les stations amont.

5) - Eusimulium latipes Mg. : elle colonise surtout le Lot et la Truyère au-dessus de $1000 \mathrm{~m}$, mais est peu abondante. Elle est associée à $P$. nigripes.

6) - Wilhelmia salopiensis (Rdw.) $=$ W. lineata $(\mathrm{Mg}$.$) : La syno-$ nymie a été établie par Crosskey et DAvies (1972). Cette espèce se rencontre à partir de St-Geniez dès que les eaux se réchauffent. Emergences : VIII.

7) - Wilhelmia equina L. : souvent en association avec l'espèce précédente, elle préfère les eaux plus chaudes. Elle a été récoltée dans le Célé à Marcilhac et sur les chaussées du Lot canalisé. Grenier (1953) mentionnait sa présence dans le Lot à Port d'Agrès. Elle n'a pu y être retrouvée. Emergences : VIII-IX. W. equina et $W$. salopiensis sont des espèces tolérantes vis-à-vis de la pollution (Rubzov 1967, CARLSSON 1967).

8) - Obuchovia auricoma $\mathrm{Mg}$. : C'est une espèce commune localement, là où le courant est fort (Tournel). Divers auteurs (GrENIER 1953, Rubzov 1959-1964, Giudicelli 1961, Zwick 1974) ont déjà noté sa préférence pour ce genre de biotope. Emergences : VIIII.

9) - Odagmia ornata $\mathrm{Mg}$. : espèce récoltée dans les ruisselets d'altitude, sur la végétation aquatique, quand le courant n'est pas très fort (Truyère). SMART (1934) mentionne déjà cette rhéophobie relative. Cette espèce, surtout d'altitude, semble n'avoir qu'une génération par an (Emergences : V-VI), bien que GRENIER (1949) pense que même au-dessus de $1000 \mathrm{~m}$ l'espèce est bivoltine. L'association $O$. ornata-W. equina citée par GrENIER (1949), n'a pu être observée. Les deux espèces ont des aires de répartition ne se chevauchant pas dans le Lot. $O$. ornata se rencontre quand l'eau a une température moyenne de $13^{\circ} \mathrm{C}$ environ, tandis que $W$. equina se trouve dans des eaux plus chaudes.

10) - Odagmia variegata $\mathrm{Mg}$. : C'est l'espèce dominante sur le Lot, dès que le débit est suffisant. Elle disparaît après Entraygues. Sa répartition semble essentiellement liée à la vitesse du courant. Phillipson (1957) a montré que cette espèce est adaptée au courant violent. C'est une espèce de moyenne altitude (de 900 à $200 \mathrm{~m}$ ), qui 
semble toutefois eurytherme. Elle forme la base des populations de Simuliidae, seules les espèces accompagnatrices varient. L'espèce semble univoltine en altitude (VIII-IX), biovoltine dès $400 \mathrm{~m}$ (V et IX).

11) - Simulium rheophilum $(\mathrm{Knoz}, 1920)=$ Odagmia monticola Fried. $=O$. obreptans Edw. : La révision de cette espèce a été faite par H. Zwick (1974). Cette espèce a son maximum d'abondance dans le Lot et la Truyère vers $900-1000 \mathrm{~m}$. La longueur de la période de vol (fin mai - début septembre) permet de supposer que l'espèce est au moins bivoltine, mais l'étalement des émergences dû à l'hétérogénéité du peuplement (GRENIER, 1949) ne permet pas d'individualiser chaque génération.

L'association $O$. variegata - S. rheophilum déjà signalée (PHILliPson 1957, Giudicelli 1961) a été maintes fois retrouvée dans le Lot. Les deux espèces ont des exigences écologiques très proches. Grenier (1949) indique que leur répartition serait influencée par la nature du substrat. Elles préféreraient les roches cristallines. Dans le Lot, les plus grandes quantités de larves ont été capturées sur des schistes ou des blocs cristallins.

12) - Simulium reptans L. : Plus euryoque que ne l'indiquent certains auteurs (SMART 1944, GRENIER 1949, KNoz 1965), cette espèce est présente dans le Lot de la source à Fumel, mais jamais abondante. Zwick (1974) note aussi la présence de populations de $S$. reptans dans le potamon. Dorogostaisky, Rubzov et Vlassenko (1935) ont récolté l'espèce en Sibérie, dans les rivières dont la température estivale n'excède pas $12^{\circ} \mathrm{C}$. L'eurycité de l'espèce semble donc confirmée. La période de vol (VII) ne concerne que les populations de la haute Truyère. Ailleurs la faible densité de nymphes ne permettait pas de donner des indications valables.

13) - Simulium argyreatum $\mathrm{Mg} .=S$. decorum Walk. $=$ S. nölleri Fried. : Cette espèce a des exigences écologiques particulières. Elle se rencontre massivement en des endroits très limités : déversoirs d'étangs et de biefs de basse altitude (GRENIER 1949). Elle pullulait au déversoir du bief de Luzech ( $100 \mathrm{~m})$; Rivosecchi (1967) l'a récoltée en Italie et elle est fréquente en Europe du Nord. Elle semble donc avoir une répartition altitudinale et latitudinale plus grande qu'on ne le supposait jusqu'à présent. La nymphose a lieu massivement en septembre. Ceci rejoint les observations de ZwICK (1974) et fait penser à une espèce univoltine. KNoz et SASINKova (1969) ont observé 2 générations par an.

14) - Tetisimulium bezzii (Corti) : Seulement deux nymphes et une larve ont été récoltées en août 1973 à St. Côme. Les caractères de cette station correspondent aux préférences de cette espèce, données par DoRIER (1963). ZWICK (1974) qualifie cette espèce de méditer- 
ranéenne, endémique. Elle a été récoltée en Afrique du Nord, Yougoslavie, Italie, Corse, Espagne, Pyrénées.

\section{4. - ETUDE DE LA REPARTITION}

L'utilisation du rapport de fréquence d'association employé par SERRA-Tosio (1963), montre que Odagmia variegata et Simulium rheophilum forme la base du peuplement simuliidien du Lot. $O$. variegata représente, à elle seule $30 \%$ des captures.

L'utilisation du pourcentage de similitude de Whittaker (1952) et WhitTaker et Fairbanks (1958) a permis de mettre en évidence des groupes de stations ayant des populations voisines (fig. 3).

Ce pourcentage tient compte de l'abondance relative des espèces (tableau I).

$$
\text { Ps }=100-0,5 \mathrm{~s}|\mathrm{x}-\mathrm{y}|
$$

$\mathrm{x}$ et $\mathrm{y}=$ abondance relative des espèces dans les stations $\mathrm{X}$ et $\mathrm{Y}$. Les discontinuités (fig. 3) mettent en évidence plusieurs groupes de stations : $a_{1}, a_{,} a_{2}, b, c$,

- Le groupement $a_{1}$ correspond aux stations au-dessus de $1000 \mathrm{~m}$. Les espèces récoltées dans ces stations sont de espèces d'altitude psychrothermes : $P$. hirtipes, $P$. rufipes, $P$. nigripes et $E$. latipes. Le manque d'altitude fait que, dans le Lot, ces espèces sont à la limite inférieure de leur répartition altitudinale et le préférendum thermique, lié à l'altitude, ne peut être déterminé pour les espèces de ces stations. La station $\mathrm{T} 1$ ressemble aussi à celles du groupe a pour ce qui concerne la vitesse du courant et le débit.

- Le plus grand nombre de stations se retrouvent dans le groupement a. Ce sont des stations d'altitude moyenne entre 350 et $950 \mathrm{~m}$. Le courant y est fort. Les stations du Dourdou, bien que d'altitude plus faible ont le même peuplement, en raison de la température et du debit voisins.

Dans ces stations, dominent les espèces très rhéophiles : $O$. variegata, S. reptans, S. rhéophilum et Ob. auricoma.

La station 7 fait partie de ce groupement car le courant y est fort en raison des lâchers du barrage de Castelnau à l'amont. On y retrouve donc les espèces rhéophiles.

Les stations de la Truyère forment le groupement $a_{2}$. Les eaux y sont plus froides que dans le Lot. L'espèce dominante est $S$. reptans accompagnée de $O$. variegata et $S$. rheophilum. A la dernière station de la Truyère le réchauffement des eaux convient à $W$. salopiensis et $W$. equina. La station $C$ (Célé) est intermédiaire entre $a_{2}$ et $b$. Les eaux y sont plus froides que dans le Lot moyen et le débit est voisin de la haute Truyère. 


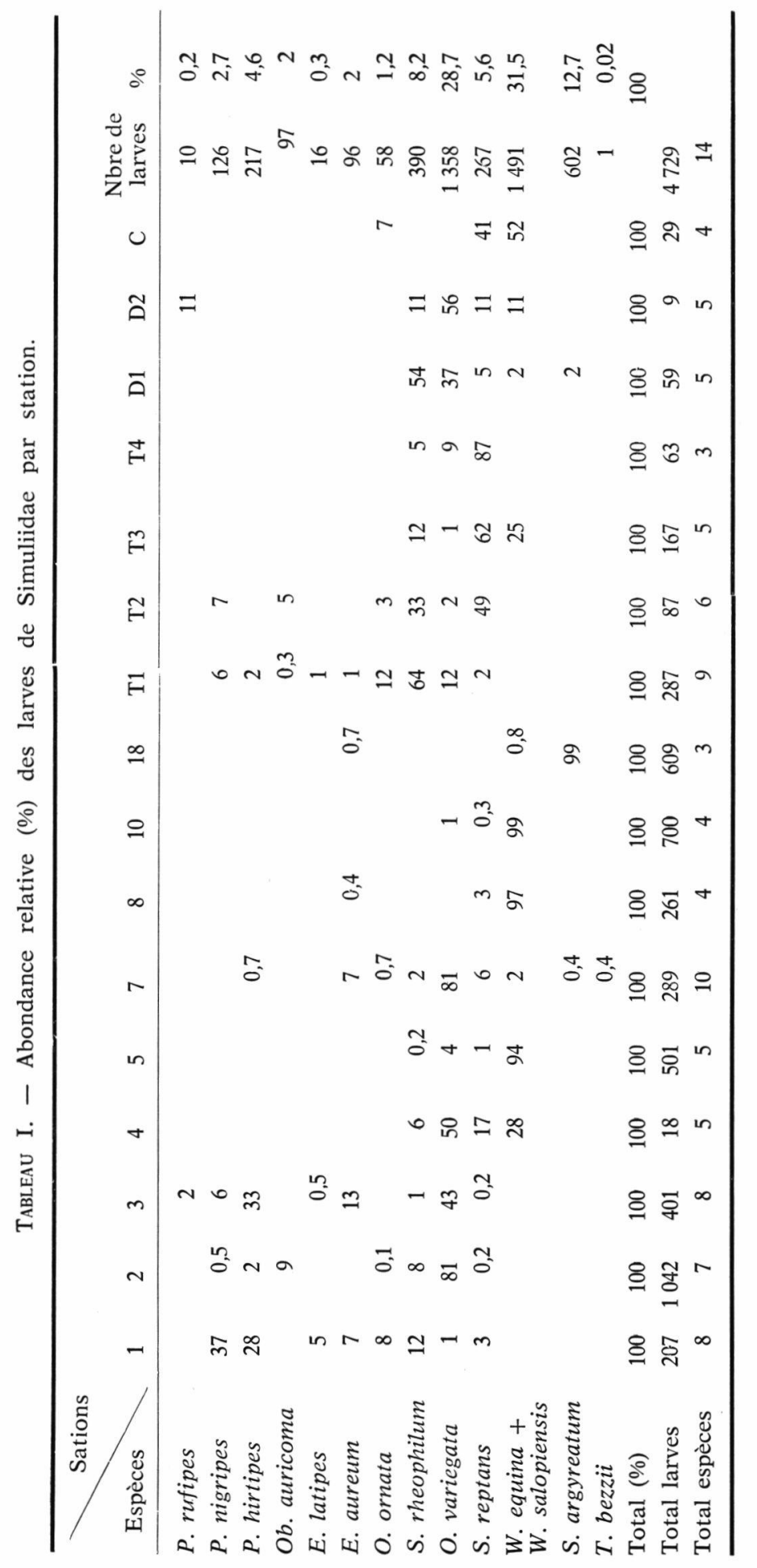




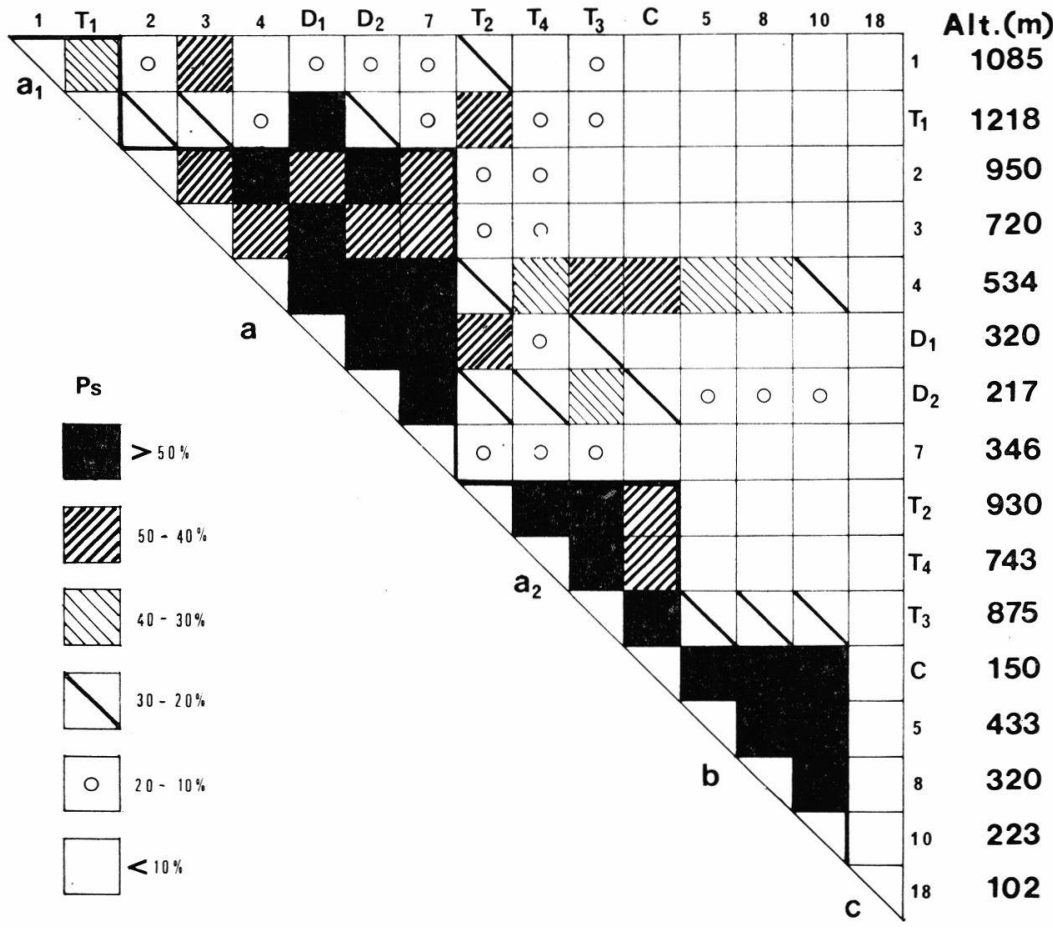

FIG. 3. - Matrice des pourcentages de similitude des stations.

- b regroupe les stations 8 et 10 du Lot, entre 320 à $220 \mathrm{~m}$ d'altitude. Le courant y est généralement faible en raison de l'élargissement du lit, mais le facteur le plus influent est le réchauffement des eaux.

L'association W. equina - W. salopiensis est caractéristique de ces stations.

La station 5, bien que d'altitude plus élevée fait partie de ce groupement. La rivière à cet endroit s'étale entraînant un courant plus faible et un plus grand réchauffement de l'eau.

- La station 18, ne ressemblant à aucune autre, est isolée en c, caractérisant les stations du Lot canalisé. Dès que le courant permet la vie des Simuliidae, $S$. argyreatum est abondant. Cependant, les Simulies n'ont pas été récoltées sur toutes les chaussées de la partie aval, certainement à cause de la remise en suspension des éléments minéraux fins par des gravières de l'amont.

Des espèces comme $E$. aureum, $O$. variegata, $S$. reptans et $S$. rheophilum, à grande répartition altitudinale, sont associées à des espèces différentes selon les stations et ne caractérisent pas un groupement comme le font : $P$. rufipes, $G b$. auricoma, Wilhelmia sp. et $S$. argyreatum. 
En résunié, il apparaît que les populations de Simuliidae sont sous l'influence combinée de la vitesse du courant, dépendant de la pente et du débit, et de la température surtout liée à l'altitude.

On remarque aussi une diminution du nombre d'espèces de l'amont vers l'aval, dans le Lot et dans la Truyère (tableau I). Dans la partie canalisée, les conditions très particulières ne conviennent qu'à un nombre restreint d'espèces dont une, $S$. argyreatum, prolifère.

\section{5. - DISCUSSION}

La présence de 9 des 14 espèces de Simuliidae du Lot, est confirmée pour la région 8 de la Limnofauna Europea (ILlies et Coll 1967). Simulium reptans et Eusimulium gr. aureum sont signalés pour la première fois dans la région 8. A l'exception de $S$. rheophilum, les autres espèces étaient déjà signalées dans la région 13 de la Limnofauna.

Les espèces du Lot sont, en général, des espèces à grande répartition européenne. C'est le cas de S. rheophilum, E. latipes, E. gr. aureum, $W$. equina, $W$ salopiensis et $O$. variegata. Cette dernière espèce offre la particularité d'être très abondante dans le Lot, alors que les auteurs signalent qu'elle est répandue mais rarement abondante (Maitland et Penney 1967 ; Neveu 1972). Le Massif Central semble avoir une faune assez homogène et commune. SERRA-Tosio (1963), dans le bassin de l'Ardèche (versant oriental du Massif Central), a mis en évidence une zonation semblable à celle du Lot. Seuls les peuplements de basse altitude diffèrent. On note que la faune simuliidienne du Lot est plus proche de celles des Alpes françaises (DorIER 1963) et autrichiennes (SUPPERER et KUTZER 1967) que de celle des Pyrénées (Grenier et Bertrand 1951; Neveu 1972 ; LavanDIER 1976). En effet, sur les 28 espèces des Pyrénées, 11 seulement sont présentes dans le Lot; ce sont des espèces à grande répartition, excepté $T$. bezzii. La présence de cette espèce montre l'influence méditerranéenne sur le sud-est du bassin; et $T$. bezzii semble être dans le Lot à la limite Nord de son aire de répartition.

La plupart des travaux sur les simulies portent sur de petits cours d'eaux (Giudicelli 1961; Maitland et Penney 1967; Supperer et Kutzer 1967 ; Neveu 1972. L'étude récente de ZwICK (1974) sur la Fulda est comparable à la présente étude car elle concerne un cours d'eau d'une certaine importance. L'étude des $500 \mathrm{~km}$ d'une rivière comme le Lot, permet d'obtenir un évantail d'espèces aux préférences écologiques variées. Cependant, le relief ne permet pas aux espèces typiquement montagnardes d'être bien représentées. En outre, les aires de répartition se chevauchant largement, les populations 
des stations diffèrent de façon surtout quantitative. Dans ces conditions, la zonation ne peut pas être établie par une méthode semblable à celle utilisée par ZWIck (1974) pour la Fulda ; il est nécessaire de tenir compte de l'abondance relative de chaque espèce.

\section{TRAVAUX CITES}

Berthelemy (C.), Laur (C.). 1975. - Plécoptères et Coléoptères aquatiques du Lot (Massif Central français). Annls. Limnol., 11 (3) : 263-285.

Bordes (J.-M.), Luchetta (J.-C.) et Rochard (Y.). 1973. - Etude d'un écosystème d'eau courante : le Lot. Thèse $3^{\circ}$ cycle Toulouse.

CARLSSON (G.). 1967. - Environmental factors influencing Blackfly populations. Bull. Wld. Hith. Org., 37 : 139-150.

Crosskey (R. W.) et Davies (L.). 1972. - The identities of Simulium lineatum

(Meigen), S. latipes (Meigen) and S. vernum Macquart (Diptera : Simuliidae). Entom. Gaz., Kettering, $23: 249-258$.

Dauta (A.). 1975. - Etude du phytoplancton du Lot. Annls. Limnol., 11 (3): $219-238$.

Davies (L.). 1968. - A key to the British species of Simuliidae (Diptera) in the larval, pupal and adult stages. Freshw. Biol. Ass. Scient. Publ., $\mathrm{N}^{\circ} 24$

Décamps (H.), Massio (J.-C.) et Darcos (J.-C.). 1976. - Variations des teneurs en matières minérales et organiques transportées dans une rivière canalisée, le Lot. Annls. Limnol. 12 (3).

Dorier (A.), Freychet (J.). 1945-1948. - Stations de larves et de nymphes de de Simulies en Dauphiné. Trav. Lab. Hydr. Pisc. Grenoble, 37-40 : 15-21.

DoRIER (A.). 1963. - Documents pour servir à la connaissance des Simuliidae du S-E de la France. Trav. Lab. Hydr. Pisc., Grenoble, 54-55 : 7-79.

Dorogostajsky (U. V.). Rubzov (I. A.) et Vlasenko (N. M.). 1935. - Notes on the taxonomy, biology and geographical distribution of Black flies in East Siberia (Simuliidae, Diptera) : Mag Parasitol. Inst. Zool. Aca. Sci. USSR, $5: 107-204$

Gagneur (J.). 1976. - Etude des Diptères du Lot et étude de la retenue de Cajarc. Thèse $3^{\circ}$ cycle, Toulouse : $189 \mathrm{p}$.

Giudicelli (J.). 1961. - Récoltes de Simuliidae (Diptera) en Corse. Etude faunistique et écologique. 86e Congr. de Soc. Sav. : 715-723.

Grenier (P.). 1949. - Contribution à l'étude biologique des Simuliides de France. Physiol. Comp. Oecol., s'Gravenhage, 1 : 165-330.

Grenier (P.). 1953. - Simuliidae de France et d'Afrique du Nord. Paris Encyclopédie Entomologique, $29: 170 \mathrm{p}$.

Grenier (P.) et BERTRAND (H.). 1951. - Récoltes de Diptères Simuliidae et Blepharoceridae dans les Pyrénées. Vie et Milieu (4) : 488-500.

Illies (J.) et Coll. 1967. - Limnofauna Europea. Fischer Verlag. Stuttgart, $\mathrm{XV}+474 \mathrm{p}$.

KNOZ (J.). 1965. - To identification of Czechoslovakian Black flies (Dipt. Sim.). Prirod. Fak. Univ. I. E. Purkyné (Biol. 2) Brno, 6 (5) : 1-54.

Knoz (J.) et Sasinkova. 1969. - Zur Kenntnis der Kriebelmücken (Simuliidae, Diptera) im Dyje-Gebiet in Morava. Fol. fac. sci. nat. Univ. Purkymanae Brunensis (Biol. 25), $10(8): 13-44$.

LaVANDier (P.). 1976. - Premières données sur la répartition des Simuliidae (Diptères, Nematocères) dans la vallée d'Aure (Hautes-Pyrénées, France). Bull. Soc. Hist. Nat., Toulouse, 112 (1-2) : 89-95.

Maitland (P.S.), Penney (M.). 1967. - The ecology of the Simuliidae in a Scottish river. J. Anim. Ecol., London, 36 : 179-206.

Neveu (A.). 1972. - Introduction à l'étude de la faune des Diptères à larves aquatiques d'un ruisseau des Pyrénées-Atlantiques : le Lissuraga. Annls. Hydrobiol., 3 (2) : 173-196.

Phillipson (J.). 1957. - The effect of current speed on the distribution of the larvae of the Black flies $S$. variegatum $\mathrm{Mg}$. and $S$. monticola Fried. Bull. ent. Res., 48 (3) : 
PodszuHN (H.). 1967. - Gattungbestimmung von europäischen Simuliidenlarven (Diptera). Gewäss. Abwass. (Düsseldorf), 44-45: 87-95.

Rivosecchi (L.). 1962. - Contributo alla conoscenza dei Simulidi Italiani III. Su qualche specie dei gruppi E. latipes (Mg.) ed E. aureum (Fries) raccolta Nell'italia centrale ed meridionale. Riv. Parassit. 23 (2) : 135-150.

Rivosecchi (L.). 1967. - I Simulidi degli Appennini. Parassitologia. Rom 9 (3) : 129-304.

Rubzov (I. A.). 1959-1964. - Simuliidae - In Lindner E. : Die Fliegen der paläarktischen Region, III (4) : 1-689.

Rubzov (I. A.). 1967. - Simuliidae. In : Illies J. Limnofauna Europaea. Fischer Verlag, Stuttgart : 340-345.

Serra-Tosio (B.). 1963. - Larves nymphes de Simuliidae (Diptera) du bassin de l'Ardèche. Trav. Lab. Hydr. Pisc. Grenoble, 54-55 : 123-142.

SMART (J.). 1944. - The british Simuliidae with keys to the species, in the adult, pupal and larval stages. Sc. Publ. Freshw. biol. Ass. Brit Emp., 9 : 1-57.

SupPERER (R.), KuTZER (E.) 1967. - Beitrag zur Kriebelmücken-Fauna (Diptera, Simuliidae) Ósterreichs. III - Die Fauna des Ybbstales. Verh. zool. bot. Ges. Wien, 107 : 25-27.

Whittaker (R. H.). 1952. - A study of summer foliage insect communities in the great Smoky Mountains. Ecol. Monogr., 2 : 1-44.

WhitTaker (R. H.) et Fairbanks (C. W.). 1958. - A study of plankton copepod communities in the Columbia basin, south eastern Washington. Ecology, $39: 46-65$.

ZWICK (H.). 1974. - Faunistischökologische und taxonomische Untersuchungen an Simuliidae (Diptera) unter besonderer Berücksichtigung der Arten des Fulda-Gebietes. Abh. Senckenberg. Naturforsch. Ges., N $N^{\circ} 533: 1-116$. 